\title{
Field-induced superconductor-to-insulator transition in Josephson-junction ladders
}

\author{
Enzo Granato \\ Laboratório Associado de Sensores e Materiais, Instituto Nacional de Pesquisas Espaciais, 12245-970 São Josẽ dos Campos, SP Brazil
}

(Received 3 August 2005; published 30 September 2005)

\begin{abstract}
The superconductor-to-insulator transition is studied in a self-charging model for a ladder of Josephson junctions in the presence of an external magnetic field. Path integral Monte Carlo simulations of the equivalent $(1+1)$-dimensional classical model are used to study the phase diagram and critical behavior. In addition to a superconducting (vortex-free) phase, a vortex phase can also occur for an increasing magnetic field and small charging energy. It is found that an intervening insulating phase separates the superconducting from the vortex phases. Surprisingly, a finite-size scaling analysis shows that the field-induced superconducting-to-insulator transition is in the KT universality class, even though the external field breaks time-reversal symmetry.
\end{abstract}

DOI: 10.1103/PhysRevB.72.104521

PACS number(s): 74.40.+k, 74.81.Fa, 73.43.Nq, 74.25.Qt
Superconductor-to-insulator transitions in Josephson junction arrays have attracted considerable interest. ${ }^{1-5}$ Such arrays can currently be fabricated in any desired geometry both in one and two dimensions ${ }^{4,5}$ with well-controlled parameters. When charging effects due to the small capacitance of the grains or junctions dominate, strong quantum fluctuations of the phase of the superconducting order parameter may drive the system into an insulating phase at zero temperature, leading to a superconductor-to-insulator transition as a function of charging energy or an external magnetic field. In two dimensions, the universality class of these transitions have already been investigated in detail numerically, ${ }^{6}$ both in relation to experiments ${ }^{3,4}$ and theoretical predictions. ${ }^{7-9} \mathrm{Nev}-$ ertheless, there are also remarkable quantum phase transition scenarios ${ }^{10}$ that can take place in a Josephson-junction ladder in a magnetic field as in Fig. 1 which have not been investigated experimentally or numerically. Interestingly, a Josephson-junction ladder provides the simplest onedimensional version of the array allowing the study of commensurability effects due to the flux lattice ${ }^{10-13}$ or charge frustration, ${ }^{14}$ in the presence of quantum fluctuations.

An important aspect of the Josephson-junction ladder in Fig. 1 is the approximate relation to the quantum sineGordon model, ${ }^{15}$ as first shown by Kardar. ${ }^{10}$ For an increasing magnetic field, there is a transition from a (vortex-free) superconducting phase to a vortex phase, where flux penetrates the ladder, in the absence of quantum fluctuations. This transition is the analog of the commensurateincommensurate transition ${ }^{16}$ described by the sine-Gordon model. For small fields, the phases in different branches of the ladder are locked to each other while in the vortex state exponentially interacting kinks (vortices) appear that unlock the phases leading to a one-dimensional vortex lattice. The inclusion of quantum fluctuations due to charging effects leads to the interesting prediction that an insulating phase should occur in the vicinity of this transition and therefore a direct transition from the (vortex-free) superconducting phase to the vortex lattice phase is not possible, even for small charging energies. So far, this remarkable collective effect resulting from small capacitances in the ladder has not been observed experimentally, or even numerically. The quantum critical behavior is also of great interest. While at the zero field the superconductor-to-insulator transition is in the Kosterlitz-Thouless universality class, ${ }^{11,17}$ the fieldinduced superconductor-to-insulator transition could be, in principle, in a different universality class, since the magnetic field breaks the time reversal symmetry and the commensurate-incommensurate transition in the sine-Gordon model is in a distinct universality class. ${ }^{15,16}$

In this work, we use path integral Monte Carlo (MC) simulations of the equivalent $(1+1)$-dimensional classical model to study the phase diagram and critical behavior of the Josephson-junction ladder. The exchange MC method (parallel tempering $)^{18}$ is used to determine more accurately the scaling behavior. It is found that an intervening insulating phase separates the superconducting from the vortex phases, as shown in Fig. 2, in agreement with Kardar's prediction. ${ }^{10}$ Thus, for an increasing field a single transition to an insulator occurs for charging energies in the range $g_{B}<g<g_{A}$ while for lower charging energies the superconductor-to-insulator transition is followed by a transition into a vortex phase. Surprisingly, a finite-size scaling analysis shows that the field-induced superconducting-to-insulator transition ac is in the KT universality class.

We consider a periodic Josephson-junction ladder, as indicated in Fig. 1, where charging effects are dominated by the capacitance to the ground of each grain, ${ }^{17}$ and described by the Hamiltonian ${ }^{10}$

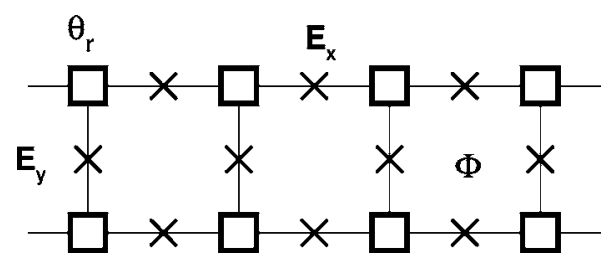

FIG. 1. A schematic representation of a periodic Josephsonjunction ladder. Superconducting grains (squares) with charging energy $E_{c}$ are coupled to the nearest neighbors by intra- $\left(E_{x}\right)$ and interchain $\left(E_{y}\right)$ Josephson couplings (crosses). The frustration $f$ corresponds to the magnetic flux through the elementary cell, $f$ $=\Phi / \Phi_{0}$, in units of the flux quantum $\Phi_{0}$. 


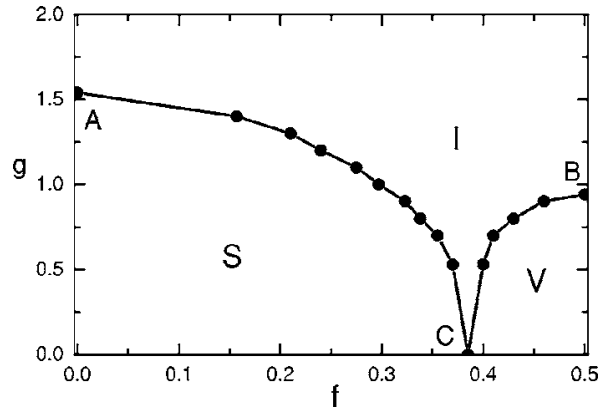

FIG. 2. Phase diagram of the ladder obtained from path integral MC simulations showing the (vortex-free) superconducting phase $(\mathrm{S})$, the insulating phase (I), and the vortex phase $(\mathrm{V})$, as a function of frustration $f$ and ratio of charging to Josephson energy $g$ $=\left(E_{c} / E_{x}\right)^{1 / 2}$ for $E_{y} / E_{x}=2$. The critical field $f_{c}$ at $g=0$ was obtained separately, by considering the ground state of the ladder in the absence of quantum fluctuations $\left(E_{c}=0\right)$. The lines are just guides to the eye.

$$
\mathcal{H}=-\frac{E_{c}}{2} \sum_{r}\left(\frac{d}{d \theta_{r}}\right)^{2}-\sum_{\left\langle r r^{\prime}\right\rangle} E_{r r^{\prime}} \cos \left(\theta_{r}-\theta_{r^{\prime}}-A_{r r^{\prime}}\right) .
$$

The first term in Eq. (1) describes quantum fluctuations induced by the charging energy $E_{c}=4 e^{2} / C$ of a non-neutral superconducting grain located at site $r$, where $e$ is the electronic charge and $C$ is the effective capacitance to the ground of the grain, while the second term is the usual Josephsonjunction coupling between nearest-neighbor grains described by phase variables $\theta_{r}$. The effect of the applied magnetic field appears through the bond variables $A_{r r^{\prime}}$ $=\left(2 \pi / \Phi_{0}\right) \int_{r}^{r^{\prime}} A d r$, where $\mathbf{A}$ is the vector potential due to the external magnetic field $\mathbf{B}$ and the gauge-invariant sum around an elementary cell of the ladder is given by $\Sigma_{r r^{\prime}}$ $=2 \pi f$, with $f=\Phi / \Phi_{0}$ defining the frustration parameter. It is sufficient to consider $f$ in the range [0, 0.5].

In order to study the effects of the magnetic field on the superconductor-to-insulator transition and the critical behavior, it is convenient to use an imaginary-time path-integral formulation of the model. ${ }^{2}$ In this formulation, the onedimensional (1D) quantum problem of Eq. (1) maps into a 2D classical statistical mechanics problem with the extra dimension corresponding to the imaginary-time direction. The time axis $\tau$ can be discretized in slices $\Delta \tau$ and the ground state energy of the quantum model corresponds to the reduced free energy of the classical model, per unit length in the imaginary time direction. After rescaling the time slices appropriately in order to get space-time isotropic couplings, the resulting classical partition function is given by $Z$ $=\operatorname{Tr}_{\{\theta\}} e^{-H}$, where the reduced classical Hamiltonian can be defined as

$$
\begin{aligned}
H= & -\frac{1}{g} \sum_{\tau, j}\left[\cos \left(\theta_{\tau, j}-\theta_{\tau+1, j}\right)\right. \\
& +\cos \left(\theta_{\tau, j}^{\prime}-\theta_{\tau+1, j}^{\prime}\right)+\cos \left(\theta_{\tau, j}-\theta_{\tau, j+1}-\pi f\right) \\
& \left.+\cos \left(\theta_{\tau, j}^{\prime}-\theta_{\tau, j+1}^{\prime}+\pi f\right)+\frac{E_{y}}{E_{x}} \cos \left(\theta_{\tau, j}-\theta_{\tau, j}^{\prime}\right)\right] .
\end{aligned}
$$

In Eq. (2), $\theta$ and $\theta^{\prime}$ denote the phases in the upper and lower branches in Fig. 1, $j$ and $\tau$ label the spatial and time directions, respectively, and the ratio of the charging energy to the Josephson coupling $g=\left(E_{c} / E_{x}\right)^{1 / 2}$ plays the role of an effective temperature in the $2 \mathrm{D}$ classical model. The equation (2) corresponds to a gauge choice where the vector potential $A$ is parallel to the ladder, with opposite values in the upper and lower branches.

We carry out MC simulations using the 2D classical Hamiltonian in Eq. (2) regarding $g$ as a temperaturelike parameter and employing the standard Metropolis' algorithm to generate the equilibrium distribution. Near the superconductor-to-insulator transition, we use the exchange MC method (parallel tempering) ${ }^{18}$ to determine more accurately the scaling behavior of various physical quantities. In fact, this method is known to reduce significantly the critical slowing down near the transition. In this method, many replicas of the system with different couplings $g$ in a range above and below the critical point are simulated simultaneously and the corresponding configurations are allowed to be exchanged with a probability distribution satisfying detailed balance. Simulations are performed in system sizes with equal spatial and time linear length $L$. This choice of the aspect ratio of the system assumes implicitly that the dynamic critical exponent $z$ characterizing the superconductorto-insulator transition is close to $z \sim 1$. In general, a quantum phase transition is characterized by intrinsic anisotropic scaling with different diverging correlation lengths $\xi$ and $\xi_{\tau}$ in the spatial and time directions, ${ }^{2}$ respectively, related by the dynamic exponent $z$ as $\xi_{\tau} \propto \xi^{z}$. Our choice is justified by the analysis discussed below, showing that the scaling behavior is in fact consistent with $z=1$.

To locate the superconductor-to-insulator transition as a function of the charging energy and magnetic field, we have performed MC calculations of the helicity modulus (phase stiffness), $\rho$ and $\rho_{\tau}$, in the spatial and time directions. In the superconducting phase these quantities should be finite, reflecting the existence of phase coherence, while in the insulating phase they should vanish in the thermodynamic limit. Figure 3(a) shows the behavior of the helicity modulus as a function of frustration for a fixed value of $g$, below the critical value corresponding to $f=1 / 2$ frustration in Fig. $2, g_{B}$ $\sim 0.96$. The helicity modulus remains finite at small fields and at fields close to $f=1 / 2$, where a superconducting phase is expected, ${ }^{12}$ but at intermediate fields reach small values that decreases with increasing system sizes. Figure 3(b) also shows the behavior of the energy fluctuation (the specific heat of the classical model) $C$ as a function of frustration showing two peaks near the two critical fields defining the region where the helicity modulus reach small values. The behavior in Figs. 3(a) and 3(b) indicate that there are two superconducting-to-insulator transitions for increasing $f$ with an intervening insulating phase corresponding to vanishing phase stiffness for large system sizes $L$. Performing the same calculations for different couplings $g$, we have constructed the phase diagram in Fig. 2 for $E_{y} / E_{x}=2$ showing three different phases separated by the transition lines $\mathrm{AC}$ and $\mathrm{BC}$ with the insulating phase extending between the superconducting vortex-free phase $\mathrm{S}$ and vortex phase $\mathrm{V}$ for $g<g_{B}$. The critical field $f_{c}$ at $g=0$ was obtained separately, by con- 

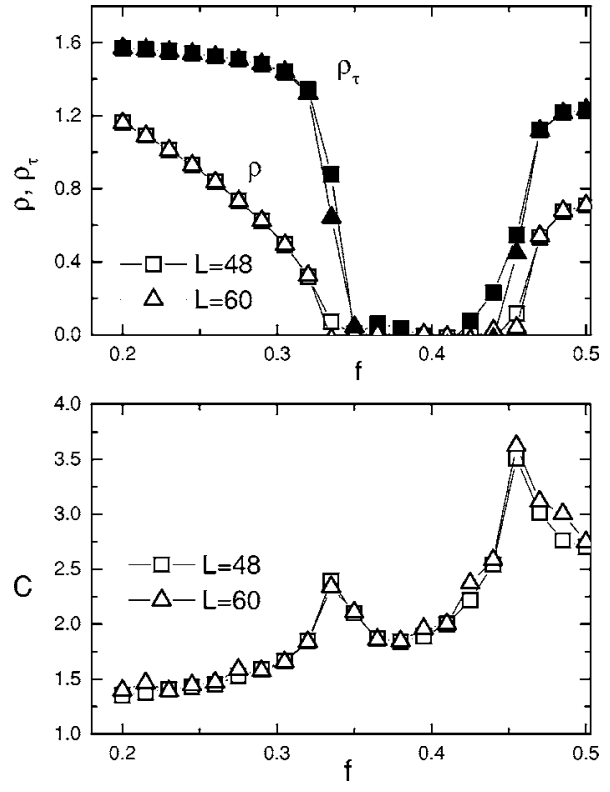

FIG. 3. Phase stiffness in spatial $\rho$ and imaginary time $\rho_{\tau}$ directions (a) and energy fluctuation $C=\left(\left\langle H^{2}\right\rangle-\langle H\rangle^{2}\right) / L^{2}$ of the equivalent classical model [Eq. (2)], as a function of frustration $f$, for a constant value of the ratio $g=0.9$. The double peaks in $C$ indicate two transitions for increasing $f$ with an intervening insulating phase corresponding to vanishing phase stiffness.

sidering the ground state of the ladder in the absence of quantum fluctuations $\left(E_{c}=0\right)$. Using simulated annealing to obtain the ground state, $f_{c}$ was determined as the value of $f$ where flux first penetrates the ladder for increasing $f$. Note that, due to the long runs required to reach proper equilibration, the lowest nonzero value of $g$ accessible within the present MC calculations is $g \sim \frac{1}{2} g_{B}$. Therefore, this result does not rule out the possibility that the two transitions lines in Fig. 2 merge at a lower value of $g$ into a single line ending at $f_{c}$, in which case a direct transition from $\mathrm{S}$ to $\mathrm{V}$ phases is possible for sufficiently small $g$. We have performed additional calculations with various ratios $E_{y} / E_{x}$ and found that the insulating phase is more clearly visible for $E_{y} / E_{x}>1.5$, while at lower values, the transition lines $\mathrm{AC}$ and $\mathrm{BC}$ are difficult to resolve at low values of $g$.

The intervening insulating phase between the superconducting phase at low fields and vortex phase at higher fields was predicted analytically in an earlier work by $\operatorname{Kardar}^{10}$ using an approximate relation of the Hamiltonian of Eq. (1) with Gaussian and sine-Gordon models describing fluctuations of the phase variables $\phi=\left(\theta^{\prime}+\theta\right) / 2$ and $\psi=\left(\theta^{\prime}-\theta\right) / 2$, respectively, where $\theta^{\prime}$ and $\theta$ denote the phases in the upper and lower branches of the ladder in Fig. 1. This approximation allows one to calculate the power-law correlation functions for $\theta$ and $\theta^{\prime}, C(r) \propto 1 / r^{\eta}$, describing the superconducting and vortex phases, using the exact results for the sineGordon model. ${ }^{15}$ However, the assumed Gaussian approximation leaves out space-time vortices that are responsible for the insulating phase, where the correlation function decays exponentially. The insulating phase was then studied by adding the effects of space-time vortices "by hand" through the usual KT criterion $\eta<1 / 4$ to determine the bound of stability of the power-law correlated phase. The results showed that the insulating phase expected for large $g$ extends all the way to $g=0$ at $f_{c}$. The phase diagram in Fig. 2 is consistent with this prediction, but as mentioned previously a direct transition between $\mathrm{S}$ and $\mathrm{V}$ phases at sufficiently small $g$ cannot be ruled out within the present calculations.

Although a KT-like stability criterion was used in Kardar's work ${ }^{10}$ to determine approximately the location of the transitions to the insulating phase in the phase diagram, the nature of the transition cannot be determined by such an argument because it is based in decoupled Gaussian and sine-Gordon models. Like in a similar analysis employing the KT criterion for the sine-Gordon model, ${ }^{19}$ applied to adsorbed layers, which also predict an intervening disordered phase near the commensurate-incommensurate transition for low-order commensurate phases, the universality class of the commensurate to disordered phase has to be determined by other methods based on symmetry considerations, mapping to a solvable model or numerical simulations.

The universality class of the field-induced superconductor-to-insulator transition along the line AC in Fig. 2 is of particular interest. At zero magnetic field $(f=0)$ the transition as a function of charging energy is in the KT universality class ${ }^{11,17}$ while at nonzero magnetic fields, in principle, a different universality class is possible since the magnetic field breaks the time-reversal symmetry. In addition, the relation of the ladder model of Eq. (1) with the quantum sine-Gordon chain ${ }^{10}$ also suggests the possibility of a universality class different from the KT transition at zero field since the superconductor-to-insulator transition in the ladder is driven by the commensurate-incommensurate transition in the sine-Gordon model, which is in a distinct universality class. ${ }^{15,16}$ In order to study the critical behavior of the transition line AC in detail we have investigated the finite-size behavior of the phase correlation lengths $\xi$ and $\xi_{\tau}$ in addition to the helicity modulus $\rho$ and $\rho_{\tau}$ near the transition line AC. The correlation lengths $\xi(L)$ and $\xi_{\tau}(L)$ in the finite-size system can be obtained from a second moment calculation using the correlation function as ${ }^{20}$

$$
\xi(L)=\frac{1}{2 \sin (k / 2)}[S(0) / S(k)-1]^{1 / 2},
$$

where $S(k)$ is given by the Fourier transform

$$
S(k)=\sum_{x}\left\langle e^{i\left(\theta_{\tau, j}-\theta_{\tau, j+x}\right.}\right\rangle e^{i k x}
$$

The wave vector $k=2 \pi / L$ is the smallest value allowed in the finite system. Similar expressions are used for the correlation length $\xi(L)_{\tau}$ in the time direction.

Figure 4 shows the finite-size behavior of the correlation length scaled by the system size $L$ near the transition line AC in Fig. 2, as a function of $g$, for a fixed value of frustration $f=0.2<f_{C}$. In the insulating phase for $g>g_{c}$, the correlation length is finite and therefore $\xi / L$ and $\xi_{\tau} / L$ decrease with system size and should vanish in the limit $L \rightarrow \infty$ while at the transition and in the superconducting phase, where the system is critical, $\xi / L$ should approach a finite value depending 
(a)
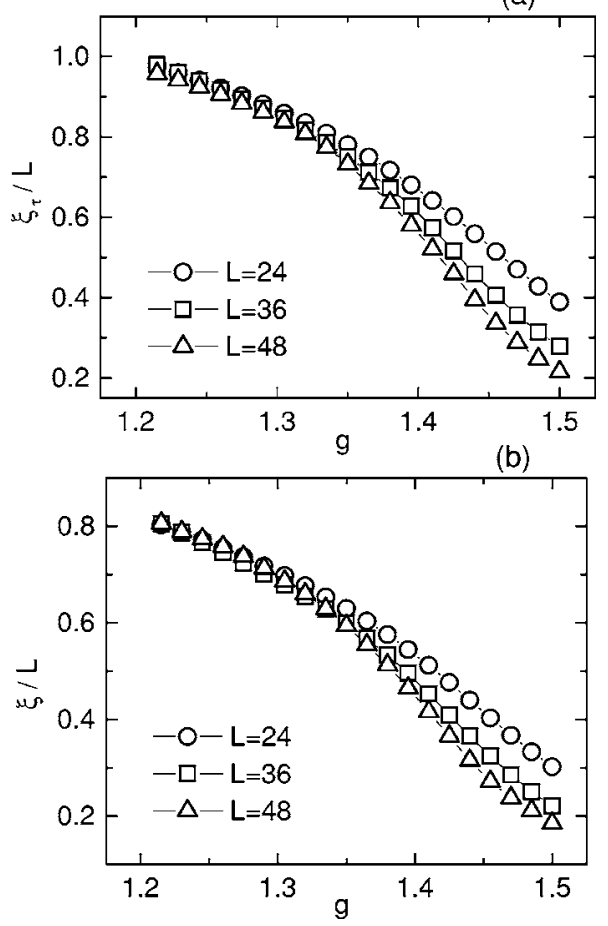

FIG. 4. Behavior of the scaled correlation length $\xi_{\tau} / L$ (a) and $\xi / L$ (b) across the superconductor-to-insulator transition line $A C$ in Fig. 2 at a fixed nonzero frustration $f=0.2$, for different system sizes $L$.

of $g$. The behavior in Figs. 4(a) and 4(b), showing that both $\xi / L$ and $\xi_{\tau} / L$ merge for low values of $g$ at approximately the same critical value $g_{c} \sim 1.3$ is consistent with a transition in
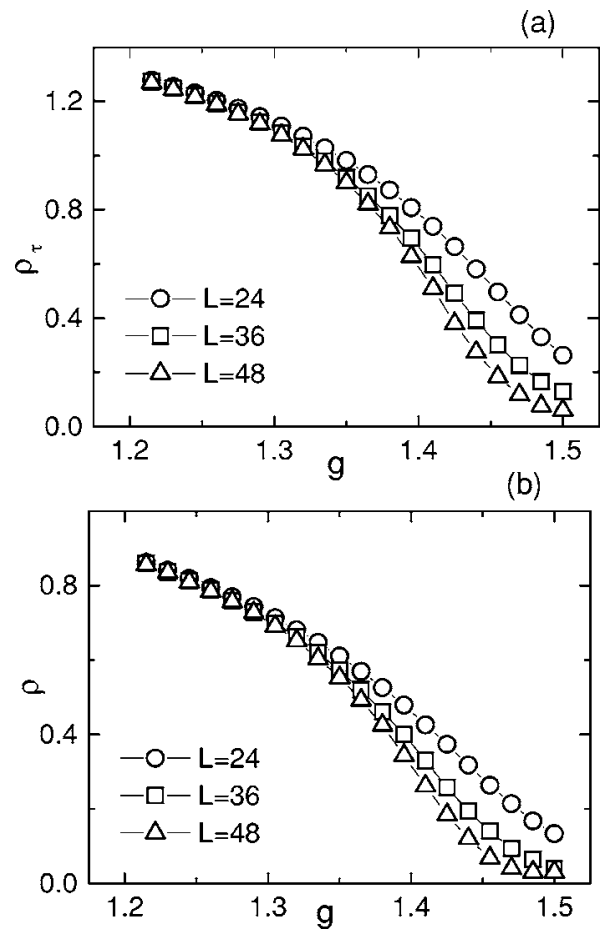

FIG. 5. Behavior of the phase stiffness $\rho_{\tau}$ (a) and $\rho$ (b) across the superconductor-to-insulator transition line AC in Fig. 2 at a fixed nonzero frustration $f=0.2$, for different system sizes $L$.

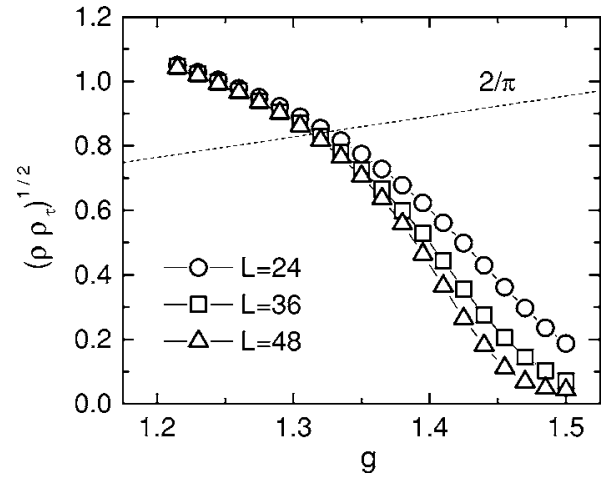

FIG. 6. Behavior of the quadratic average of the phase stiffness $\left(\rho \rho_{\tau}\right)^{1 / 2}$ across the superconductor-to-insulator transition line AC in Fig. 2 at a fixed nonzero frustration $f=0.2$, for different system sizes $L$. A dotted line corresponds to the universal jump prediction for a KT transition.

the KT universality class, where the correlation length diverges exponentially $\xi \propto e^{b /\left|g-g_{c}\right|^{1 / 2}}$. In general, for a powerlaw correlation length $\xi \propto\left|g-g_{c}\right|^{-1 / 2}$, the dimensionless quantity $\xi / L$ is expected to cross at the transition for different system sizes ${ }^{20}$ and satisfy the scaling relation $\xi / L=G[(g$ $\left.\left.-g_{c}\right) L^{1 / \nu}\right]$ near the transition. Then, the slopes $s(L)$ of the curves $\xi / L$ for different $L$ are determined by the critical exponent $\nu$ as $s(L)=(\partial / \partial g)(\xi / L) \propto L^{1 / \nu}$, evaluated near $g_{c}$. The exponential correlation length of the KT transition corresponds to $\nu \rightarrow \infty$, which implies that $s(L)$ should be $L$ inde-

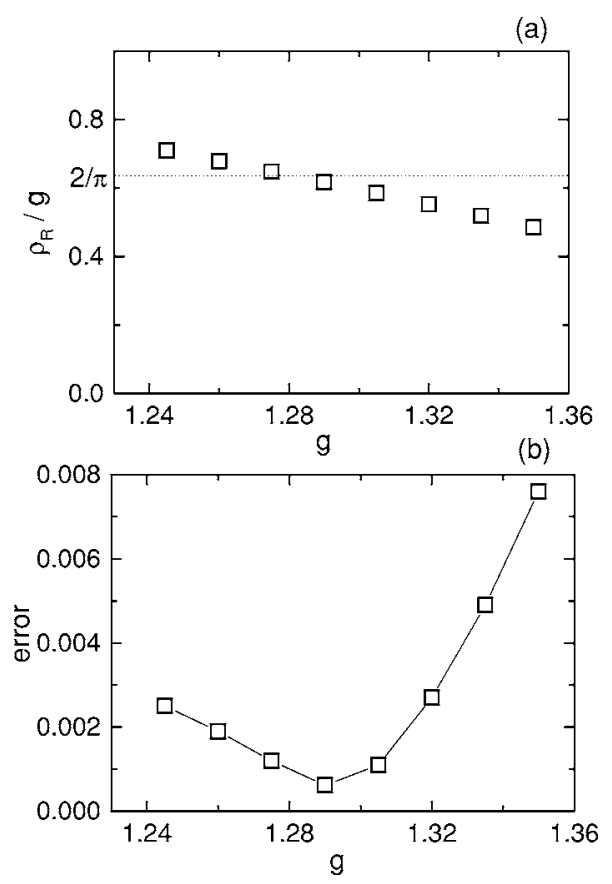

FIG. 7. Numerical fit of the phase stiffness $\bar{\rho}$ according to the Weber and Minnhagen scaling relation with two free parameters for system sizes $L=6,8,12,16,18,24$, at different couplings $g$ across the superconductor-to-insulator transition. (a) The estimate of the jump $\rho_{R} / g_{c}$ from the fitting parameter and (b) error estimate of the fit. The location of the minimum in (b) determines the critical coupling $g_{c}$. The dotted line in (a) is the value of the universal jump for a KT transition. 
pendent and therefore the curves $\xi / L$ for different system sizes should merge at the critical point, as, in fact, observed in Fig. 4.

The finite-size behavior of the helicity modulus $\rho$ and $\rho_{\tau}$ is also consistent with a KT transition. As shown in Fig. 5, $\rho$ and $\rho_{\tau}$ also merge for decreasing $g$ at approximately the same critical $g_{c}$. At the transition and in the superconducting phase, where the system is critical, these quantities should scale as ${ }^{2,6} \rho \propto L^{2-d-z}$ and $\rho_{\tau} \propto L^{z-d}$, in $d$ spatial dimensions. Since $\rho_{\tau}$ and $\rho$ in Figs. 5(a) and 5(b) approach finite values, independent of $L$ in this regime, the dynamic exponent is $z=1$ for the present case, where $d=1$. This value of the dynamic exponent $z$ means that the line of Gaussian fixed points can be made isotropic, even though the phase stiffness, $\rho$ and $\rho_{\tau}$, are different in the spatial and time directions. In fact, by a suitable rescaling of the coordinates in the spatial and time directions, the stiffness of the renormalized isotropic Gaussian model can be written as $\bar{\rho}=\left(\rho \rho_{\tau}\right)^{1 / 2}$. If the transition is in the KT universality class, then a universal jump ${ }^{21}$ is expected for $\bar{\rho}$ as a function of $g$ at the transition given by $\bar{\rho}=2 / \pi g$. Figure 6 shows that the finite-size behavior of $\bar{\rho}$ appears consistent with the expected universal jump for the phase stiffness. For each system size, the intersection of the data with the dotted line representing the universal jump prediction provides an upper bound estimate of the critical coupling. A more quantitative determination of the critical coupling and the corresponding jump in the phase stiffness can be obtained by the Weber and Minnhagen scaling analysis, which has been introduced before as an accurate method of locating the critical coupling and the universal jump of the KT transition for the ordinary XY model..$^{22}$ The phase stiffness at the transition should have a logarithmic correction given by ${ }^{22}$

$$
\rho(L)=\rho_{R}\left(1+\frac{1}{2} \frac{1}{\ln L+C}\right),
$$

where $C$ is an undetermined constant and the universal jump implies $\rho_{R}=(2 / \pi) g_{c}$. Using data for different system sizes, the best numerical fit according to this scaling form performed for different values of the coupling $g$ can then be used to locate the transition and determine the jump. Treating both $\rho_{R}$ and $C$ as free parameters, we have performed this fit using data of the quadratic-averaged phase stiffness $\bar{g}$ for smaller system sizes $L \leqslant 24$, where the data is more accurate. As shown in Fig. 7, the value of the jump near the location of the minimum in the fitting error for different couplings $g$, is indeed consistent with the value expected for a KT universality class.

In conclusion, we have studied the superconductor-toinsulator transition in a ladder of Josephson junctions under an applied magnetic field by path integral MC methods. We found clear numerical evidence in the phase diagram of an intervening insulating phase between the superconducting and vortex phases, in good agreement with an earlier prediction by Kardar. ${ }^{10}$ In addition, we find that the field-induced superconducting-to-insulator transition is in the KT universality class. In the vortex phase, commensurability of the vortex lattice and the ladder ${ }^{11,13}$ will strongly depend on the frustration parameter $f$. Since Josephson-junction arrays can currently be fabricated in any desired geometry and with well-controlled parameters, our numerical evidence of the intermediate insulating phase and the universality class of the transition should be interesting enough to motivate experiments in these intriguing systems.

This work was supported by Fundação de Amparo à Pesquisa do Estado de São Paulo (FAPESP, Proc. no. 03/00541$0)$.
${ }^{1}$ R. Fazio and H. van der Zant, Phys. Rep. 555, 235 (2001).

${ }^{2}$ S. L. Sondhi, S. M. Girvin, J. P. Carini, and D. Sahar, Rev. Mod. Phys. 69, 315 (1997).

${ }^{3}$ L. J. Geerligs, M. Peters, L. E. M. de Groot, A. Verbruggen, and J. E. Mooij, Phys. Rev. Lett. 63, 326 (1989).

${ }^{4}$ H. S. J. van der Zant, L. J. Geerligs, and J. E. Mooij, Europhys. Lett. 119, 541 (1992).

${ }^{5}$ W. Kuo and C. D. Chen, Phys. Rev. Lett. 87, 186804 (2001).

${ }^{6}$ H. Lee and M.-C. Cha, Phys. Rev. B 65, 172505 (2002); M.-C. Cha and S. M. Girvin, ibid. 49, 9794 (1994); M. C. Cha, M. P. A. Fisher, S. M. Girvin, M. Walling, and A. P. Young, Phys. Rev. B 44, 6883 (1991).

${ }^{7}$ M. P. A. Fisher, G. Grinstein, and S. M. Girvin, Phys. Rev. Lett. 64, 587 (1990); M. P. A. Fisher, ibid. 65, 923 (1990).

${ }^{8}$ E. Granato and J. M. Kosterlitz, Phys. Rev. Lett. 65, 1267 (1990).

${ }^{9}$ R. Fazio and G. Schön, Phys. Rev. B 43, 5307 (1991).

${ }^{10}$ M. Kardar, Phys. Rev. B 33, 3125 (1986).

${ }^{11}$ E. Granato, Phys. Rev. B 42, 4797 (1990).

${ }^{12}$ E. Granato, Phys. Rev. B 45, 2557 (1992).
${ }^{13}$ E. Orignac and T. Giamarchi, Phys. Rev. B 64, 144515 (2001).

${ }^{14}$ M.-S. Choi, M. Y. Choi, T. Choi, and S. I. Lee, Phys. Rev. Lett. 81, 4240 (1998).

${ }^{15}$ F. D. M. Haldane, J. Phys. A 15, 507 (1982).

${ }^{16}$ V. L. Pokrovsky and A. L. Talapov, Theory of Incommensurate Crystals, Soviet Science Reviews (Harwood, New York, 1984).

${ }^{17}$ R. M. Bradley and S. Doniach, Phys. Rev. B 30, 1138 (1984).

${ }^{18}$ K. Hukushima and K. Nemoto, J. Phys. Soc. Jpn. 65, 1604 (1996); E. Marinari and G. Parisi, Europhys. Lett. 19, 451 (1992).

${ }^{19}$ F. D. M. Haldane, P. Bak, and T. Bohr, Phys. Rev. B 28, 2743 (1983).

${ }^{20}$ H. G. Ballesteros, A. Cruz, L. A. Fernandez, V. Martin-Mayor, J. Pech, J. J. Ruiz-Lorenzo, A. Tarancon, P. Tellez, C. L. Ullod, and C. Ungil, Phys. Rev. B 62, 14237 (2000).

${ }^{21}$ D. R. Nelson and J. M. Kosterlitz, Phys. Rev. Lett. 39, 1201 (1977).

${ }^{22}$ H. Weber and P. Minnhagen, Phys. Rev. B 37, 5986 (1988). 